\title{
A Goal Matching Service for Facilitating Public Collaboration Using Linked Open Data
}

\author{
Shun Shiramatsu ${ }^{1}$, Teemu Tossavainen ${ }^{1,2}$, Tadachika Ozono ${ }^{1}$, \\ and Toramatsu Shintani ${ }^{1}$ \\ 1 Graduate School of Engineering, Nagoya Institute of Technology, Japan \\ \{siramatu, ozono, tora\}@nitech.ac.jp \\ 2 School of Science, Aalto University, Finland \\ teemu.tossavainen@aalto.fi
}

\begin{abstract}
Inter-organizational collaboration in the public sphere is essentially important to address sustainability problems in contemporary regional societies. To facilitate public collaboration, we are developing a Web application for sharing public issues and their solutions as public goals. Since participating in abstract or general goals is more difficult than concrete or specific ones, our system provides a functionality to break down individual public goals into concrete subgoals. Our Web application, GoalShare, is based on a linked open dataset of public goals that are linked with titles, participants, subgoals, related issues, related articles, and related geographic regions. GoalShare recommends public goals and users on the basis of similarity calculations taking into account not only surficial and semantic features but also contextual features extracted from subgoals and supergoals. We conducted experiments to investigate the effects of contextual features in subgoals and supergoals. Moreover, we conducted a trial workshop with GoalShare in Ogaki city to improve system design through actual use.
\end{abstract}

Keywords: linked data, civic tech, public involvement, concern assessment, text mining.

\section{Introduction}

Contemporary human societies confront problems with sustainability, e.g., increased consumption of resources, polarization into rich and poor 11, and catastrophic risks of the disasters due to climate change [2]. Inter-organizational collaboration in the public sphere is essentially important to improve the sustainability of regional society because the sustainability problems are relevant to various stakeholders and are intricately interrelated. There have recently been public collaborations with social networking services (SNSs) to address regional issues in Japan, especially after the Great East Japan Earthquake in 2011 [34]. It is, however, difficult to find potential collaborators who have similar public concerns with SNSs because most SNSs emphasize the aspect of real-time information sharing and lack of functions to share "who are trying to address

E. Tambouris et al. (Eds.): ePart 2014, LNCS 8654, pp. 114-127, 2014.

(C) IFIP International Federation for Information Processing 2014 
particular issues and what kinds of approaches they take". When many groups focus on similar social issues, collaboration among them reduces nonproductive conflicts and facilitates constructive consensus-building or social innovation.

We have designed linked open data (LOD) of social issues and their solutions as public goals, which enabled us to calculate similarities between public goals [5]. Moreover, we have implemented a prototype of a Web application, called GoalShare (ゴオルシェア in Japanese), to match citizens or groups who have similar goals to facilitate public collaboration and open innovation [6]. For this purpose, user-generated LOD of public goals are linked with personal identifiers of existing SNSs and geographical identifiers of existing geographical LODsets by the GoalShare system. In this paper, we discuss its applicability to public collaboration in the real world and empirical investigations based on an actual dataset of public goals gathered in Japanese regional societies.

The matching function needs to take into consideration two conditions to inter-organizationally match people with their collaboration potential.

(a) Similarity of issues focused on or goals aimed at.

(b) Complementarity of required skills, abilities, or resources.

This paper especially focuses on condition (a) as a first step. Matching with condition (b) can be formulated as a two-sided matching problem that is often modeled on the basis of the game theory [7. We do not limit the matching function to two-sided matching because many citizens, who have similar aims like those in condition (a), can be candidate collaborators one after another in the process of solving social issues.

We designed a hierarchical data model for goal description to break down an abstract goal into concrete subgoals in our previous studies [5]. Our data model could be regarded as a simplification of a prerequisite tree (PRT) in the theory of constraint (TOC) pioneered by Goldratt [8]. Data on issues and goals are linked in our model with personal information such as participants, with geographical information such as related regions, and with temporal information such as deadlines to specialize them in public participation. We expect three effects by sharing LOD in a hierarchical goal structure.

(i) Providing Hints As To Which Activity can be Contributed To. It is difficult to determine how to participate in or contribute to an abstract or general goal, whereas it is easier to determine whether a concrete subgoal can be contributed to or not because the grain-size of concrete subgoals is closer to actual actions or activities than that of abstract goals.

(ii) Sharing Context for Negotiations of Collaboration Among Groups. Even groups that have similar objectives occasionally conflict with one another because subgoals are sometimes difficult to be agreed on even if the final goal is generally agreed on. Visualizing their goal hierarchies enables them to compare the structures, to check which part is conflictive, and to try to find a common ground. 
(iii) Sophisticating Calculations of Similarities between Goals. To calculate similarities, the hierarchical structure enables us to use not only surficial or semantic features in textual goal descriptions but also contextual features such as supergoals or subgoals.

Although the hierarchical structure of goals needs to be input through deliberative processes, we need to gather public goals and issues from regional societies at the early stages of service operation. We assumed three ways of inputting public goals at an early stage.

(1) Input by Goal Owners. Identical people who have goals personally input and publish them to disclose their own activities and explore potential collaborators.

(2) Input by Interviewers. Researchers, social workers, or government officers ask citizens about issues and input their responses. They also create solutions as goal data and find potential contributors.

(3) Input by Third Parties Using References. Citizens extract public goals planned by key personalities from fragmentary news articles and share the hierarchical structure of goals to increase the transparency of social movements or projects,

Input through deliberative processes will occur after "seed data" are gathered in these three ways.

\section{Linked Open Data of Public Issues and Goals}

We designed LOD of public issues and goals in our previous research [5] and specialized them through developing the GoalShare [6] outlined in Figure 1, The classes socia:Goal and socia: Issue have titles and descriptions as textual content, and are linked with a related geographical region by dcterms:spatial,

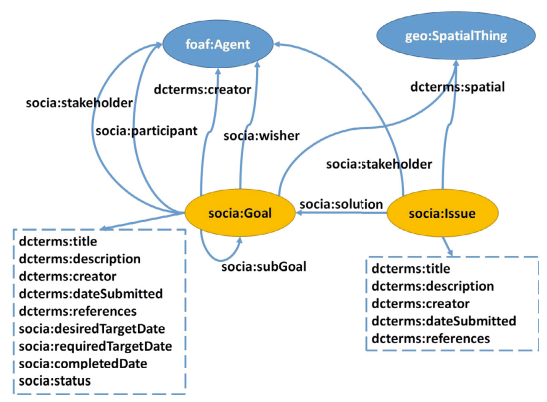

Fig. 1. Data model for describing public issues and goals 
a creator by dcterms:creator, and created date by dcterms: dateSubmitted. The socia: Goal class especially has links to subgoals by socia: subGoal to represent the hierarchical structure and links to participants by socia:participant to represent a team of collaborators.

In our previous research, we constructed a dataset consisting of 657 public goals for recovery and revitalization from the Great East Japan Earthquake [5]. The goals were manually extracted from 96 news articles and two related documents on the Web.

After preliminary investigations using the dataset, the data model has been specialized for implementing GoalShare. The prototype of GoalShare has an RDF triple store and a SPARQL endpoint powered by OpenLink Virtuosd 1 . These 657 goals for earthquake recovery and new issues/goals created by GoalShare users are stored in the RDF triple store. The user-generated goals are linked with personal identifiers with existing SNSs (Facebook and Twitter) and geographical identifiers with existing geographical LODs (GeoNames and GeoLOD $\left.{ }^{3}\right)$. These links to the data hub potentially contribute to the interoperability of our dataset, which results from the LOD approach. The dataset needs to be interoperable to facilitate collaboration among existing groups on Facebook.

The goals and issues in our RDF triple store have openly been published via our SPARQL endpoint, http: //collab.open-opinion .org/sparql. For example, the following SPARQL query returns the following RDF triples that represent a public goal for measures against earthquakes in Nagoya city.

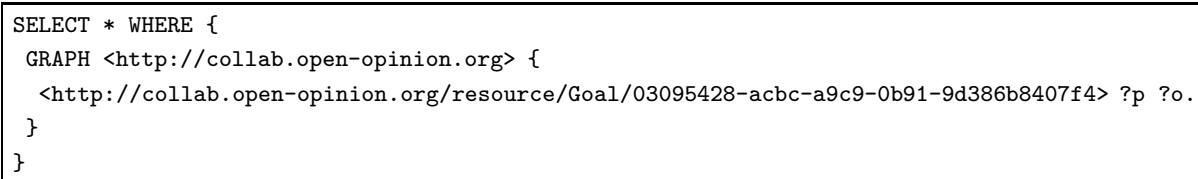

<http://collab.open-opinion.org/resource/Goal/03095428-acbc-a9c9-0b91-9d386b8407f4> rdf:type socia:Goal;

dcterms:title "Measures against the coming earthquake at Nankai Trough"; dcterms:creator <http://collab.open-opinion.org/resource/Person/f5b8e09ac510>; dcterms:spatial <http://geolod.ex.nii.ac.jp/resource/EqBQEA>; dcterms: dateSubmitted "2013-12-09T14:00:00+09:00" ^^xsd:dateTime; dcterms:references <http://wWw.nikkei.com/article/DGXNASFD3000F_Q3A530C1CN8000/>; socia: subGoal <http://collab.open-opinion.org/resource/Goal/a0d4b89fe3f3>; socia: subGoal <http://collab.open-opinion.org/resource/Goal/9d386b8407f4>; socia:desiredTargetDate "2015-01-01" ^ xsd:date.

\section{Development of GoalShare}

\subsection{Design of User Interface}

There is a screenshot of the user interface of the current prototype of GoalShare 6] in Figure 2, It has a multilingual user interface (English, Japanese, and Finnish)

\footnotetext{
1 http://virtuoso.openlinksw.com/

2 http://www.geonames.org/

3 http://geolod.ex.nii.ac.jp/
} 


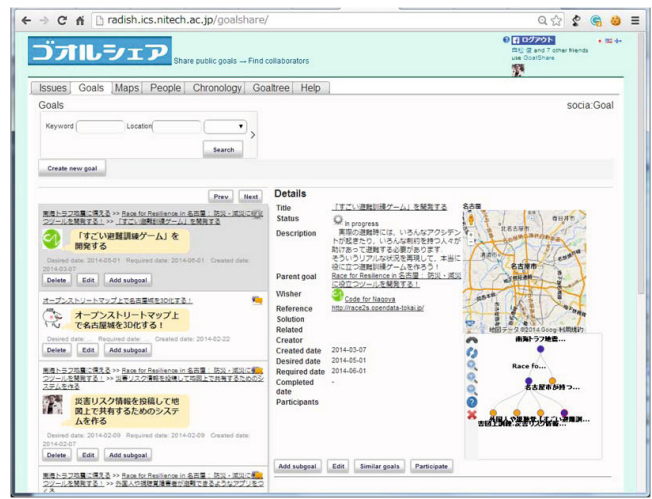

Fig. 2. User Interface of GoalShare

and is available via http://radish.ics.nitech.ac.jp/goalshare/. Although it currently supports logins with Facebook accounts, users also can input issues or goals anonymously without logins. Since we assumed that recent public goals would be suitable for understanding overviews of social movements or projects, the "Goals" tab is first opened in Figure 2 when users access the above URL. In the "Goals" tab, the most recently created goals are listed on the left of the figure. Details on the selected goal are on the right of the figure. The detailed section includes a map of related regions linked with the selected goal and a hierarchical visualization of subgoals and supergoals of the selected goal.

The detailed section contains buttons for "Add subgoal", "Edit", "Similar goals", and "Participates". Users can break down the selected goal into more concrete subgoals with the "Add subgoal" button and find goals similar to the selected goal with the "Similar goals" button. The method we used for calculating similarities is described in a later subsection. Moreover, users can register themselves as participants of the selected goal with the "Participants" button. We assumed that such functions effectively increased motivation for planning solutions and contributed to resolving issues.

Although sharing public goals on the "Goals" tab is essential to facilitate collaboration among motivated citizens, gathering issues on the "Issues" tab should occur prior to constructing goal structures because sharing issues can be the first cue to resolving public issues. Additionally, inputting issue data is easier for most citizens than creating hierarchical goal structures because public issues can be noticed in daily life. Inputting public goals as solutions requires thinking and planning through deliberative processes. Hence, the "Issues" tab provides an "Add as a goal" button to create an abstract goal as a solution to selected issues.

The validity of these prototype designs needs to be tested, verified, and improved through actual use by citizens to enable practical application in real-world societies. 


\subsection{Goal Generation from Twitter}

We implemented a module for creating issues and goals in GoalShare from Twitterf. Twitter is a social media microblog service where users can post and read short text messages called Tweets. The length of a tweet is limited to 140 characters, and it can contain user defined topics known as hashtags that are marked with a "\#" prefix. Tweets can be searched with hashtags, which allow individual Tweets to be grouped to conversations. One common way to use Twitter is in events, like conventions, so that there is a commonly known hashtag that participants append to tweets so that all tweets forms a conversation concerning that event.

In our research, Twitter presents multiple advantages. First, Twitter offers a HTTP API5 for using the service programmatically. It enables us to search Tweets by topic, geographic location, and by tweeting users. Second, we can easily extract the textual content and while the text is written in natural language, Tweets have a clear convention for marking topics. In addition, Tweets can contain links to other internet materials that can be used as references in the generation of goal data. Moreover, users have to have accounts for posting Tweets, which we can use in GoalShare. The concepts of users are important in GoalShare. Users related goal information is used in the function for recommending potential collaborators for identifying similar types of users.

The implemented module searches periodically Tweets concerning predefined sets of hashtags. The first hashtag set is a collection of topics, like "\#goalshare", that defines monitored conversations. We defined the second set as action keywords, like "\#goal" and "\#issue", which are used to determine the user's intention to create an item in questions in GoalShare. With this method, we can generate goals from specific conversations and label the generated GoalShare items accordingly.

When an unprocessed Tweet with these hashtags is encountered, the module extracts the following data from the Tweet: Tweet's unique identification code, text content, hashtags, and information about the tweeting user. First, the module creates a user for the system with Twitter information, like a name and an avatar image. The user is visually represented by the avatar in GoalShare. Second, the module uses the GoalShare HTTP API to generate an item, a goal or an issue, in GoalShare with the extracted Tweet data.

In future work on the Twitter export module, we plan to add automatic suggestion of issues without the user needing to use specific hashtags. Since a training corpus of social issues and goals is needed for this research plan, we intend to gather Tweets on issues and goals with the hashtags as positive examples in the corpus. Such a corpus can also openly be published as LOD to contribute to the research field of natural language processing.

\footnotetext{
${ }^{4}$ http://twitter.com

${ }^{5}$ https://dev.twitter.com/
} 


\section{Calculating Similarity between Goals}

\subsection{Methodology}

In our previous research, we proposed a method of calculating the similarities between goals [5, which took into account surficial features in textual content, latent semantic features obtained with Latent Dirichlet Allocation (LDA) 9], and contextual features of linked subgoals. Additionally, we used an implementation of the hierarchical Dirichlet process-LDA (HDP-LDA) 6 in the training phase of a topic model [10].

However, we found that supergoals also contained contextual information by analyzing a dataset of 657 goals on recovery from the Great East Japan Earthquake. Moreover, the previous method overemphasized contextual features when there were few linked subgoals and supergoals. After this analysis, we improved the method of calculation [6]. The similarity between public goals $g_{i}$ and $g_{j}$ can be calculated using bag-of-features vectors bof $\left(g_{i}\right)$ and bof $\left(g_{j}\right)$.

$$
\begin{aligned}
& \operatorname{sim}\left(g_{i}, g_{j}\right)=\frac{\operatorname{bof}\left(g_{i}\right) \cdot \operatorname{bof}\left(g_{j}\right)}{\left\|\operatorname{bof}\left(g_{i}\right)\right\|\left\|\operatorname{bof}\left(g_{j}\right)\right\|}, \\
& \operatorname{bof}(g)=\frac{1-\gamma(g)}{\left\|\operatorname{bof}_{\text {self }}(g)\right\|} \operatorname{bof}_{\text {self }}(g)+\frac{\gamma(g)}{\left\|\operatorname{bof}_{\text {cntxt }}(g)\right\|} \operatorname{bof}_{\text {cntxt }}(g), \\
& \operatorname{bof}_{\text {self }}(g)=\frac{\alpha}{\|\operatorname{tfidf}(g)\|} \operatorname{tfidf}(g)+\frac{\beta}{\|\operatorname{lda}(g)\|} \operatorname{lda}(g), \\
& \operatorname{tfidf}(g)=\left(\begin{array}{c}
\operatorname{tfidf}\left(w_{1}, g\right) \\
\vdots \\
\operatorname{tfidf}\left(w_{|W|}, g\right) \\
0 \\
\vdots \\
0
\end{array}\right) \in \mathbb{R}^{|W|+|Z|}, \operatorname{lda}(g)=\left(\begin{array}{c}
0 \\
\vdots \\
0 \\
\mathrm{p}\left(z_{1} \mid g\right) \\
\vdots \\
\mathrm{p}\left(z_{|Z|} \mid g\right)
\end{array}\right) \in \mathbb{R}^{|W|+|Z|}, \\
& \operatorname{bof}_{\mathrm{cntxt}}(g)=\sum_{\text {subg } \in \operatorname{sub}(g)} \operatorname{bof}_{\text {sub }}(s u b g)+\sum_{\text {supg } \in \sup (g)} \operatorname{bof}_{\text {sup }}(\text { supg }), \\
& \operatorname{bof}_{\text {sub }}(g)=d_{\text {sub }}\left(\operatorname{bof}_{\text {self }}(g)+\sum_{s u b g \in \operatorname{sub}(g)} \operatorname{bof}_{\text {sub }}(s u b g)\right), \\
& \operatorname{bof}_{\text {sup }}(g)=d_{\text {sup }}\left(\operatorname{bof}_{\text {self }}(g)+\sum_{\text {supg } \in \sup (g)} \operatorname{bof}_{\text {sup }}(\text { supg })\right) \text {, and } \\
& \gamma(g)=\text { upper }_{\mathrm{cntxt}} \cdot \tanh \left(k \cdot\left\|\operatorname{bof}_{\mathrm{cntxt}}(g)\right\|\right) .
\end{aligned}
$$

This is where $g$ denotes a public goal, and bof $(g)$ denotes a bag-of-features vector of $g$. Here, $\operatorname{sub}(g)$ denotes a set of subgoals of $g, \sup (g)$ denotes a set of supergoals of $g, d_{\text {sub }}$ and $d_{\text {sup }}$ denote decay ratios when recursively tracking subgoals and supergoals respectively. The $w \in W$ denotes a term, $z \in Z$ denotes

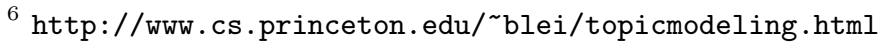


a latent topic derived by a latent topic model [9], and $\operatorname{tfidf}(w, g)$ denotes the TF-IDF, i.e., the product of term frequency and inverse document frequency, of $w$ in a title and a description of $g$. Parameter upper $_{\text {cntxt }}$ denotes an upper limit of the weight of $\operatorname{bof}_{\text {cntxt }}(g), \alpha+\beta=1$, and $0 \leq \alpha, \beta$, upper ${ }_{\text {cntxt }}, d_{\text {sub }}, d_{\text {sup }} \leq 1$.

The two types of bag-of-feature vectors, bof $_{\text {self }}(g)$ and bof cntxt $_{\text {ch }}(g)$, are newly defined to incorporate contextual information not only from subgoals but also from supergoals. The bof $f_{\text {self }}(g)$ represents a bag-of-features vector only extracted from target goal $g$ and bof $_{\text {cntxt }}(g)$ represents a contextual bag-of-features vector extracted from subgoals and supergoals.

To recommend similar goals, a pair of goals $g_{i}$ and $g_{j}$ satisfying $\operatorname{sim}\left(g_{i}, g_{j}\right)>$ $\theta_{g}$ is linked by the property schema:isSimilarTo that has been defined by schema.org7. Note that there are goal pairs that are not suitable to link by using schema: isSimilarTo despite large similarities, i.e., goal pairs that are already linked by the socia:subGoal property or goal pairs that have the same wishers or the same participants because such goal pairs already have collaborative relationships and do not need to be matched or recommended. Hence, such cases are filtered out when linking similar goals by using the schema:isSimilarTo property.

\subsection{Experiments: Contextual Effects of Subgoals and Supergoals}

The proposed method of calculating the similarities between goals requires parameters $\alpha, \beta, d_{\text {sup }}, d_{\text {sub }}$, upper ${ }_{\text {cntxt }}$, and $k$ to be empirically set. Although quantitative optimization of the parameter set needs training data consisting of

Table 1. Similarity ranking of goal pairs where $\alpha=\beta=0.5, d_{\text {sup }}=d_{\text {sub }}=0.4$, upper $_{\text {cntxt }}=0.5$, and $k=0.7$

\begin{tabular}{|c|c|c|c|}
\hline \multicolumn{2}{|c|}{\begin{tabular}{|l|l|} 
Rank & Similarity \\
\end{tabular}} & \multicolumn{2}{|c|}{ Pair of goal titles (translated from Japanese) } \\
\hline 1 & 0.998 & Selling ginkgoes & $\begin{array}{l}\text { Selling "Fukushima Organic Cotton } \\
\text { Babe" }\end{array}$ \\
\hline 2 & 0.901 & Forming a new political party & $\begin{array}{l}\text { Describing the process for forming a } \\
\text { new political party }\end{array}$ \\
\hline 3 & 0.820 & $\begin{array}{l}\text { Completing projects for quake recov- } \\
\text { ery }\end{array}$ & Securing quake recovery budgets \\
\hline 4 & 0.815 & $\begin{array}{l}\text { Promoting recognizable recovery in } \\
\text { Miyako city }\end{array}$ & $\begin{array}{l}\text { Conducting lessons on disaster pre- } \\
\text { vention at elementary schools in } \\
\text { Miyako city }\end{array}$ \\
\hline 5 & 0.797 & $\begin{array}{l}\text { Ensuring employment in Yamada- } \\
\text { machi }\end{array}$ & Ensuring employment \\
\hline 6 & 0.778 & $\begin{array}{l}\text { Tree-planting ceremony in praying } \\
\text { for quake recovery }\end{array}$ & Securing quake recovery budgets \\
\hline 7 & 0.776 & $\begin{array}{l}\text { Appropriate uses of quake recovery } \\
\text { budgets }\end{array}$ & Securing quake recovery budgets \\
\hline 8 & 0.769 & $\begin{array}{l}\text { Sending public donations for quake } \\
\text { recovery }\end{array}$ & Securing quake recovery budgets \\
\hline 9 & 0.768 & $\begin{array}{l}\text { Expanding targeted areas for support } \\
\text { throughout Fukushima prefecture }\end{array}$ & $\begin{array}{l}\text { Supporting victims who were evacu- } \\
\text { ated outside of targeted area for sup- } \\
\text { port }\end{array}$ \\
\hline 10 & 0.766 & Decontamination of radiation & $\begin{array}{l}\text { Certifying decontamination contrac- } \\
\text { tors }\end{array}$ \\
\hline
\end{tabular}

${ }^{7}$ http://schema.org/Product 
positive examples (pairs of similar goals suitable for matching or recommendations) and negative examples (goals pairs unsuitable for matching or recommendations), it is not easy to construct such training data because clear definitions of suitability for matching or recommendations are not obvious and are difficult.

As preliminary experiments, we respectively varied $d_{\text {sup }}$ and $d_{\text {sub }}$ to qualitatively investigate the effects of taking into account supergoals and subgoals. A subset of 100 goals chosen from the LOD of goals for earthquake recovery were used in the experiments. The parameter set as the basis for reference before varying them was $\alpha=\beta=0.5, d_{\text {sup }}=d_{\text {sub }}=0.4$, upper $_{\text {cntxt }}=0.5$, and $k=0.7$. In this parameter setting, the decay ratios for supergoals and subgoals, $d_{\text {sup }}$ and $d_{\text {sub }}$, took the same value of 0.4 . The top ten similarity rankings of goal pairs for the reference parameter setting are listed in Table 1. These ten pairs were not greatly affected by the contextual features and decay ratios $d_{\text {sup }}$ and $d_{\text {sub }}$ because they shared the same vocabularies. The bof $f_{\text {self }}(g)$ consisting of surficial and semantic features should be close in each pair. Thus, the top ten pairs were not drastically changed when varying $d_{\text {sup }}$ and $d_{\text {sub }}$. To qualitatively investigate the effects of supergoals and subgoals as textual features, we observed goal pairs whose ranks were drastically raised by increasing the decay ratios. Tables 2 and 3

Table 2. Goal pairs whose rank is raised by emphasizing supergoals, i.e., increasing $d_{\text {sup }}$ from 0.4 to 0.8

\begin{tabular}{|c|c|c|c|c|}
\hline Rank gain & $\mathrm{nk}$ & larity & Pair of goal titles (tra) & nslated from Japanese) \\
\hline 54 & 16 & 0.738 & Saving victims' unborn children & $\begin{array}{l}\text { Supporting victims who were evacu- } \\
\text { ated outside targeted area for sup- } \\
\text { port }\end{array}$ \\
\hline 36 & 68 & 0.645 & $\begin{array}{l}\text { Limiting use of quake recovery bud- } \\
\text { gets to recovery and relief for victims }\end{array}$ & $\begin{array}{l}\text { Supporting victims who were evacu- } \\
\text { ated outside of targeted area for sup- } \\
\text { port }\end{array}$ \\
\hline 24 & 28 & 0.723 & $\begin{array}{l}\text { Enhancing concretized policies based } \\
\text { on Victims' Protection Law }\end{array}$ & $\begin{array}{l}\text { Committee for concretizing Victims' } \\
\text { Protection Law organizes public fo- } \\
\text { rum for victims across nation }\end{array}$ \\
\hline 22 & 59 & 0.659 & $\begin{array}{l}\text { Setting up contact lines for Victims } \\
\text { Protection Law }\end{array}$ & $\begin{array}{l}\text { Supporting victims who were evacu- } \\
\text { ated outside of targeted area for sup- } \\
\text { port }\end{array}$ \\
\hline 18 & 17 & 0.737 & $\begin{array}{l}\text { Citizen participation to decontami- } \\
\text { nate radiation }\end{array}$ & $\begin{array}{l}\text { Speeding up decontamination of ra- } \\
\text { diation }\end{array}$ \\
\hline
\end{tabular}

Table 3. Goal pairs whose rank is raised by emphasizing subgoals, i.e., increasing $d_{\text {sub }}$ from 0.4 to 0.8

\begin{tabular}{|r|r|r|l|l|}
\hline Rank gain & Rank & Similarity & \multicolumn{2}{|c|}{ Pair of goal titles (translated from Japanese) } \\
\hline \hline 69 & 49 & 0.691 & Supplying aid for relief of victims & $\begin{array}{l}\text { Organizing events for friendship ex- } \\
\text { changes between evacuated victims } \\
\text { and local residents }\end{array}$ \\
\hline 57 & 40 & 0.709 & Recovery in Iwate prefecture & $\begin{array}{l}\text { Tree-planting ceremony in praying } \\
\text { for quake recovery }\end{array}$ \\
\hline 56 & 32 & 0.721 & Recovery in Iwate prefecture & $\begin{array}{l}\text { Completing projects for quake recov- } \\
\text { ery }\end{array}$ \\
\hline 53 & 31 & 0.724 & Recovery in Iwate prefecture & $\begin{array}{l}\text { Promoting recognizable recovery in } \\
\text { Miyako city }\end{array}$ \\
\hline
\end{tabular}


respectively show goal pairs whose ranks are greatly raised when $d_{\text {sup }}$ becomes 0.8 and $d_{\text {sub }}$ becomes 0.8 . "Rank gain" represents how the rank was raised by increasing the decay ratio and "Rank" represents the raised rank. Whereas Table 2 shows that increasing $d_{\text {sup }}$ affected relatively concrete goals and the highly affected ranks could be raised to higher than the 20th rank, Table 3 shows that increasing $d_{\text {sub }}$ affected abstract goals such as "Recovery in Iwate prefecture" and "Supplying aid for relief of victims" and the highly affected ranks could not be raised to higher than the 30th rank. These results indicate that emphasizing contextual features in supergoals is more appropriate than emphasizing them in subgoals because concrete goals are more suitable for recommendations than abstract ones.

\section{Trial Workshop for Citizens}

To improve the system design of GoalShare through actual use, a trial workshop was held on Dec. 19, 2013 in Ogaki city in Gifu prefecture in Japan. The trial workshop was organized as part of a series of events called "Open data cafe" managed by CCL In\&. Twelve citizens participated in the workshop. There is an illustration provided to participants, which explains the structure of an issue and its solution as hierarchical goals, in Figure 3(a). Participants were divided into three groups. They first wrote regional issues on sticky notes and selected one issue though debate. They then hierarchically structured the goals to solve the selected issue and then input the issue and goal hierarchy into the GoalShare prototype.

After the trial use, participants responded to the three statements in a questionnaire about the service concept of GoalShare.

S1. After GoalShare is officially launched, I want to use this service.

S2. After public issues and goals are accumulated in GoalShare, social movements will be easier to understand.

S3. After public issues and goals are accumulated in GoalShare, participating in or contributing to solving regional issues will be easier, even for people unfamiliar with regional contexts.

The results from the questionnaire are shown in Figure 4, which indicates that two-thirds of participants agreed with S1 and S2 and over $80 \%$ of participants agreed with S3. Moreover, participants returned positive feedback suggestions to improve the design of the prototype. For example, they suggested incorporating group accounts to find civic groups and attaching tags to issues/goals to represent concerns or topics. We are planning to continuously organize such events to improve the participatory design process of GoalShare.

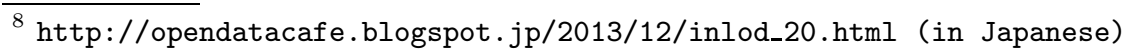



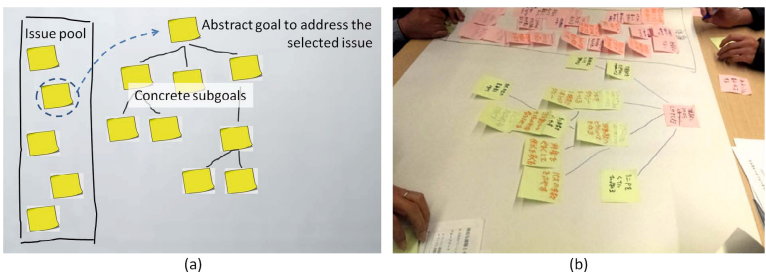

Fig. 3. (a) Illustration for workshop participants and (b) actual issues/goals written by participants

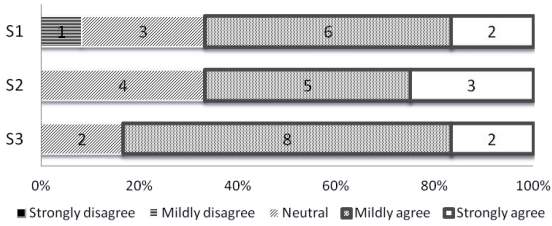

Fig. 4. Results from questionnaire after workshop in Ogaki city

\section{Related Work and our Research Context}

\subsection{Project Management}

Goals in the research field of project management are commonly structured as hierarchies by subdividing goals into subgoals. Instances of such structures are seen in the Thinking Process of the Theory of Constraint (TOC) 11 and the Work Breakdown Structure (WBS) in the Project Management Body of Knowledge (PMBOK) [12. Although these models are generally used for project management within an organization, some researchers have applied them to public sector problems. TOC, especially, has been tried to be applied to recovery from disasters 1314. Logic Models are also commonly used in the public sector for managing participatory program planning [15]. Logic Models are also used to deal with public issues and goals because building a logic model first requires issue statements and goal statements [16].

There are many Web applications for sharing tasks and managing projects, e.g., Trelld9, Cyboze Live10, and Backlog 11 . Flying Logi 12 is a software for struc-

\footnotetext{
${ }^{9}$ http://trello.com/

${ }^{10}$ http://cybozulive.com/

${ }^{11}$ http://www. backlog.jp/

12 http://flyinglogic.com/
} 
turing goal hierarchies based on TOC. However, they do not support functions for sharing goals on finding potential collaborators in public spheres. The originality of our study is dealing with goal hierarchies as open data and utilizing them to facilitate public collaboration.

\subsection{Civic Tech}

Open data technology has recently had a great affinity for the civic tech sector, i.e., technical communities for creating participatory improvements to governmental services using information technology. The development process for new tools through public collaboration such as "hackathon" events can be shared as public goals and related issues. Civic tech sector has been activated in Japan since 2013. Within this social context, the Code for Japan 13 was organized by reference to the Code for America 14 in 2013, and many hackathon events also have been organized. Continuous developments after the event were not very frequent in the hackathon in Japan, because development team members were not so many and were often holiday volunteers. We are planning to apply GoalShare to such problems, i.e., we have assumed that sharing issues and goals dealt with by civic tech communities would enable the development projects to find potential collaborators and to continue to solve the issues solving through software development. We developed a goal generation function using Twitter to be used in such civic tech events. The function can use hashtags specified by hackathon events because such events often announce their specific hashtags to participants.

\subsection{Structured Argumentation}

Structuring and visualizing argumentation effectively support eParticipation [17]. There are currently several tools for structuring and visualizing argumation, i.e., Evidence Hub [18, MIT Deliberatorium [19], etc.These tools typically produce "box and arrow" diagrams in which premises and conclusions are formulated as statements [20]. The hierarchical structures of public goals created by GoalShare users can be regarded as types of such diagrams. Moreover, the hierarchy of public goals can be a hub of argumentation networks because deliberative argumentation is needed for negotiation processes between potential collaborators by them comparing their goal hierarchies. Such argumentation data need to be linked with a shared goal hierarchy to ensure openness when they collaboratively conduct their shared plan. Developing a function for deliberation in the negotiation process based on goal hierarchies is an important future work.

\section{Conclusion}

We developed GoalShare, which is a prototype goal matching service in the public sphere. It was implemented as a Web application to match citizens who

\footnotetext{
13 http://code4japan.org/

14 http://codeforamerica.org/
} 
had similar public goals. Our system was based on an LODset of public issues and goal hierarchies, which was openly published via a SPARQL end point. The interoperability of dataset, due to the LOD approach, is required to facilitate collaboration among existing groups in existing SNSs. We formulated a similarity metric between public goals on the basis of bag-of-features vectors consisting of surficial features, latent semantic features, and contextual features. To determine the optimal parameters in our formulation, empirical investigations using an actual dataset were needed. We conducted experiments to quantitatively investigate what effects the contextual features of supergoals and subgoals had by using actual public goals about disaster recovery in Japan. The results indicate that the contextual features in supergoals should be emphasized more than those in subgoals. Moreover, we held a trial workshop for citizens and obtained positive feedback from participants.

We are planning to develop a function to support deliberative argumentation for negotiation processes between potential collaborators by comparing their goal hierarchies and a function to match citizens who have complementary skill set or resources in future work. Improvements to GoalShare will be conducted with the help of user feedbacks in trial workshops or hackathon events.

Acknowledgments. We greatly appreciate support by the Ogaki office of CCL Inc. for organizing the trial workshop of GoalShare. This work was supported by a Grant-in-Aid for Young Scientists (B) (No. 25870321) from JSPS.

\section{References}

1. Motesharrei, S., Rivas, J., Kalnay, E.: Human and Nature Dynamics (HANDY): Modeling inequality and use of resources in the collapse or sustainability of societies. Ecological Economics 101, 90-102 (2014)

2. Shi, P., Li, N., Ye, Q., Dong, W., Han, G., Fang, W.: Research on integrated disaster risk governance in the context of global environmental change. International Journal of Disaster Risk Science 1(1), 17-23 (2010)

3. Yamamoto, K.: Volunteer activities in time of disaster in japan's highly information-oriented society. Journal of Earth Science and Engineering 3(3), 190-202 (2013)

4. Yamaguchi, M., Maguth, B.: Using social networking in the social studies for global citizenship: A case study of japan's 3:11 quake. In: Proceedings of Society for Information Technology \& Teacher Education International Conference 2012, pp. 3260-3287 (2012)

5. Shiramatsu, S., Ozono, T., Shintani, T.: Approaches to assessing public concerns: Building linked data for public goals and criteria extracted from textual content. In: Wimmer, M.A., Tambouris, E., Macintosh, A. (eds.) ePart 2013. LNCS, vol. 8075, pp. 109-121. Springer, Heidelberg (2013)

6. Tossavainen, T., Shiramatsu, S., Ozono, T., Shintani, T.: Implementing a system enabling open innovation by sharing public goals based on linked open data. In: Ali, M., Pan, J.-S., Chen, S.-M., Horng, M.-F. (eds.) IEA/AIE 2014, Part II. LNCS, vol. 8482, pp. 98-108. Springer, Heidelberg (2014) 
7. Roth, A.E., Sotomayor, M.A.O.: Two-Sided Matching: A Study in Game-Theoretic Modeling and Analysis. Econometric Society Monographs, vol. 18. Cambridge University Press, Cambridge (1990)

8. Kim, S., Mabin, V.J., Davies, J.: The theory of constraints thinking processes: retrospect and prospect. International Journal of Operations \& Production Management 28(2), 155-184 (2008)

9. Blei, D.M., Ng, A.Y., Jordan, M.I.: Latent Dirichlet Allocation. Journal of Machine Learning Research 3, 993-1022 (2003)

10. Teh, Y., Jordan, M., Beal, M., Blei, D.: Hierarchical dirichlet processes. Journal of the American Statistical Association 101(476), 1566-1581 (2006)

11. AGI: The theory of constraints and its thinking processes - a brief introduction to toc, http://www.goldratt.com/pdfs/toctpwp.pdf (2009)

12. PMI: A Guide to the Project Management Body of Knowledge, 4th edn. Project Management Institute (2008)

13. Cheng, J., Shigekawa, K., Meguro, K., Yamazaki, F., Nakagawa, I., Hayashi, H., Tamura, K.: Applying the toc logistic process to clarify the problem schemes of near-field earthquake in tokyo metropolitan area. Journal of social safety science (11), 225-233 (2009) (in Japanese)

14. Ohara, M., Kondo, S., Kou, T., Numada, M., Meguro, K.: Overview of social issues after the great east-japan earthquake disaster - part 3 of activity reports of 3.11net tokyo. SEISAN KENKYU 63(6), 749-754 (2011) (in Japanese)

15. McLaughlin, J.A., Jordan, G.B.: Logic models: a tool for telling your programs performance story. Evaluation and program planning 22(1), 65-72 (1999)

16. Innovation Network: Logic model workbook, http://www.innonet.org/client_docs/File/logic_model_workbook.pdf (2005)

17. Benn, N., Macintosh, A.: Argument visualization for eparticipation: towards a research agenda and prototype tool. In: Tambouris, E., Macintosh, A., de Bruijn, H. (eds.) ePart 2011. LNCS, vol. 6847, pp. 60-73. Springer, Heidelberg (2011)

18. De Liddo, A., Buckingham Shum, S.: The Evidence Hub: Harnessing the collective intelligence of communities to build evidence-based knowledge. In: 6th International Conference on Communities and Technologies, Large-Scale Idea Management and Deliberation Systems Workshop (2013)

19. Iandoli, L., Klein, M., Zolla, G.: Enabling online deliberation and collective decision making through large-scale argumentation: A new approach to the design of an internet-based mass collaboration platform. International Journal of Decision Support System Technology 1(1), 69-92 (2009)

20. van den Braak, S.W., van Oostendorp, H., Prakken, H., Vreeswijk, G.A.W.: A critical review of argument visualization tools: Do users become better reasoners? In: Workshop Notes of the ECAI-2006 Workshop on CMNA, pp. 67-75 (2006) 